\title{
Therapiekonzepte bei niedrig-malignen Lymphomen unter Berücksichtigung der derzeit laufenden Studien
}

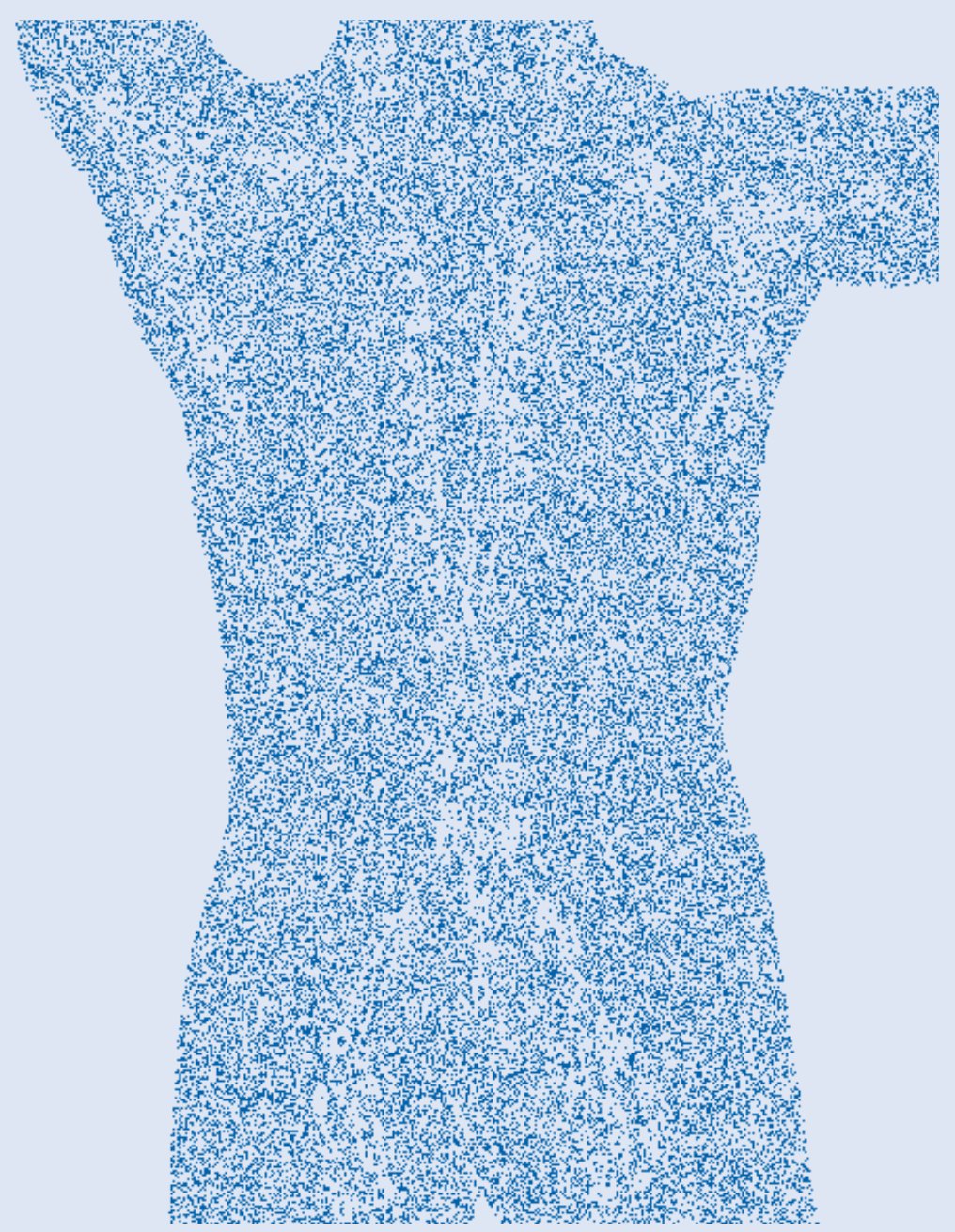




\section{Aktuelle Therapiestudien der Deutschen Studiengruppe Niedrig Maligne Lymphome bei Patienten in fortgeschrittenen Stadien}

\author{
M. Unterhalt; W. Hiddemann, München
}

Trotz zunehmender Einblicke in die Biologie und Pathogenese niedrig-maligner Non-Hodgkin-Lymphome (NHL) sind die therapeutischen Möglichkeiten bei diesen meist in fortgeschrittenen Krankheitsstadien diagnostizierten Erkrankungen begrenzt. Ein kuratives Potential etablierter zytostatischer Kombinationsregime ist bisher nicht $\mathrm{zu}$ erkennen. Aus diesem Grund existiert bislang keine allgemein akzeptierte Standardtherapie für niedrig-maligne NHL in den Stadien III und IV. Aktuelle Empfehlungen und Studienkonzepte variieren auch weiterhin von einer primär abwartenden Haltung («watch and wait») über wenig intensive zytoreduktive Therapieschemata mit Chlorambucil und Kombinationen von Cyclophosphamid, Vincristin, Prednison (COP) bis hin zum Einsatz von hochdosierten Kombinationsregimen wie M-BACOD oder Pro-MAZE-MOPP.

Neben unterschiedlichen zytostatischen Regimen wurde von mehreren Gruppen auch der Einsatz von Interferonalpha (IFN- $\alpha)$ geprüft. Dabei wurde übereinstimmend in verschiedenen Therapiestudien eine Verlängerung der Remissionszeiten durch den Einsatz von IFN- $\alpha$ beobachtet. Der Einschluß von IFN- $\alpha$ in eine initiale Chemotherapie mit einer anthracyclinhaltigen Kombination führte sogar zu einer Verlängerung der Gesamtüberlebenszeit.

Erste Ergebnisse mehrerer Phase-II-Studien weisen darauf hin, daß langdauernde Krankheitsfreiheit bei niedrigmalignen Lymphomen auch in fortgeschrittenen Krankheitsstadien möglicherweise durch die Kombination von Ganzkörperbestrahlung und Chemotherapie, gefolgt von autologer Knochenmark- oder peripherer Stammzelltransplantation, erreicht werden kann. Wegen des Mangels an Alternativen wird diese Vorgehensweise zum Teil bereits von einzelnen hämatologischen Zentren außerhalb kontrollierter Studien eingesetzt, insbesondere bei jüngeren Patienten. Dabei muß jedoch beachtet werden, daß bislang keine prospektiv randomisierten Studienergebnisse vorliegen, die den Vorteil des Verfahrens im Vergleich zu einer konventionellen Chemotherapie, insbesondere in Kombination mit einer Interferon-Erhaltungstherapie, demonstriert haben. Dieser Vorbehalt erscheint insofern von hoher Relevanz, als mehrere Berichte über eine erhöhte Inzidenz therapieassoziierter sekundärer Leukämien und myelodysplastischer Syndrome nach längerer Verlaufszeit erschienen sind. Die bisher vorliegenden Daten lassen nur retrospektive Vergleiche mit historischen Kontrollgruppen bei kleinen Fallzahlen zu und sind damit in ihrer Aussagekraft erheblich limitiert.

Vor diesem Hintergrund begann die Deutsche Studiengruppe Niedrig Maligne Lymphome 1996 mit einer prospektiv randomisierten Studie, in der die myeloablative Radio-Chemotherapie mit nachfolgender Stammzelltransplantation mit der Standard-IFN- $\alpha$-Erhaltung nach erfolgreicher initialer zytoreduktiver Chemotherapie verglichen wird. Nach Einschluß von 288 Patienten ergab die Zwischenauswertung im April dieses Jahres einen signifikanten Vorteil für den Transplantationsarm in bezug auf das krankheitsfreie Intervall. Dieses Ergebnis ist jedoch nicht Anlaß zum Beenden der Randomisation, da derzeit offenbleibt, ob sich die Verlängerung des krankheitsfreien Intervalls auch in eine längere Überlebenszeit übertragen wird. Aus diesem Grund wird die entsprechende Studie in unveränderter Weise fortgesetzt und als Endpunkt die Evaluation der Überlebenszeit definiert.

Die Studie wurde jedoch aufgrund der vorliegenden Daten zur Effektivität der beiden geprüften zytoreduktiven zytostatischen Induktionsschemata im Bereich der initialen Behandlung modifiziert. Der prospektiv randomisierte Vergleich der bisherigen Standardtherapie von Mitoxantron, Chlorambucil, Prednison (MCP) versus Cyclophosphamid, Hydroxydaunorubicin, Vincristin, Prednison (CHOP) ergab einen deutlichen Vorteil für CHOP bezogen auf die Gesamtremissionsrate als auch auf die Toxizität. Aus diesem Grund wurde die primäre zytoreduktive Chemotherapie geändert.

Die Modifikation des Studienkonzeptes geht der Frage nach, ob die Effektivität von CHOP durch die Kombination mit einem Anti-CD20-Antikörper (Rituximab) verbessert werden kann. Rituximab (Mab Thera ${ }^{\circledR}$ ) ist ein gentechnisch hergestellter monoklonaler Antikörper, der gegen das B-Lymphozyten-Oberflächenantigen CD20 gerichtet ist und mit hoher Affinität an CD20-positive Zellen bindet. Das CD20-Antigen wird von B-Zell-Vorläufern, reifen B-Zellen und vielen malignen B-Zell-Lymphomen exprimiert, nicht jedoch von Stammzellen und Plasmazellen. Der zytotoxische Wirkungsmechanismus des Antikörpers beruht auf einer komplementvermittelten Lyse von B-Zellen. Weiterhin wird zusätzlich eine Aktivierung der antikörperabhängigen zellulären Zytotoxizität vermutet. Als weitere potentielle Wirkmechanismen werden neben der Induktion der Apoptose eine Blockierung 
des $\mathrm{G}_{1} \mathrm{~S}$-Übergangs und eine gesteigerte Phosphorylierung zellulärer Proteine diskutiert.

In einer von April 1995 bis März 1996 durchgeführten multizentrischen Studie der Deutschen Studiengruppe Niedrig Maligne Lymphome konnte durch alleinige Therapie mit Anti-CD20 eine Gesamtansprechrate von $48 \%$ bei rezidivierten Patienten mit niedrig-malignem bzw. follikulärem NHL erzielt werden.

Ziel der aktuellen Studien der Deutschen Studiengruppe Niedrig Maligne Lymphome ist es, diese neuen Therapieoptionen sowohl in der Primärtherapie wie auch bei rezidivierten Patienten randomisiert zu prüfen. Zielgruppe der Studien sind Patienten im Alter über 18 Jahre, die entsprechend der aktualisierten Kiel-Klassifikation ein zentroblastisch-zentrozytisches oder zentrozytisches Lymphom oder lymphoplasmozytoides bzw. lymphoplasmozytisches Immunozytom in den fortgeschrittenen Stadien III oder IV aufweisen und die Kriterien der Therapiebedürftigkeit erfüllen. Diese bestehen in einer BSymptomatik, einer Beeinträchtigung der normalen Hämatopoese, in großen Lymphommassen («bulky disease») oder einer raschen Lymphomprogredienz.

Im Rahmen der initialen zytoreduktiven Therapie soll in den laufenden Therapiestudien überprüft werden, ob die zusätzliche Gabe von Anti-CD20 das initiale Therapieergebnis signifikant verbessern kann.

Bei unvorbehandelten Patienten soll dabei als initiale zytoreduktive Chemotherapie das CHOP-Schema [Adriamycin $50 \mathrm{mg} / \mathrm{m}^{2}$ i.v. Tag 1, Cyclophosphamid $750 \mathrm{mg} / \mathrm{m}^{2}$ i.v. Tag 1, Vincristin $1,4 \mathrm{mg} / \mathrm{m}^{2}$ (maximal 2,0 mg absolut) i.v. Tag 1, Prednison $100 \mathrm{mg} / \mathrm{m}^{2}$ per os Tage 1-5] zum Einsatz kommen. Entsprechend den bisherigen Erfahrungen der Studiengruppe ist bei initialem Einsatz dieses Schemas eine signifikante Tumorreduktion (>50\% der Lymphommasse) bei zirka $85 \%$ der Patienten zu erwarten, während eine komplette Remission bei etwa $20 \%$ der Patienten erreicht wird. Unvorbehandelte Patienten sollen daher vor Beginn der Therapie für eine Gabe von Anti-CD20 + CHOP versus alleinige CHOPTherapie randomisiert werden (Abb.1).

Bei Patienten, bei denen nach 4 Zyklen eine komplette Remission oder aber nach 6 Zyklen mindestens eine partielle Remission erreicht ist, soll wie bisher randomisiert die Wertigkeit von IFN- $\alpha$-Erhaltung und Stammzelltransplantation geprüft werden. Hierbei werden Patienten, die jünger als 60 Jahre sind und keine Kontraindikation für eine myeloablative Radio-Chemotherapie und Stammzelltransplantation aufweisen, für Stammzelltransplantation versus IFN- $\alpha$-Erhaltung $(3 \times 5$ Mio. E/Woche $)$ randomisiert. Ziel dieses Studienabschnitts ist es, die Bedeutung der myeloablativen Radio-Chemotherapie in erster



Abb. 1. Therapie bei Erstdiagnose.

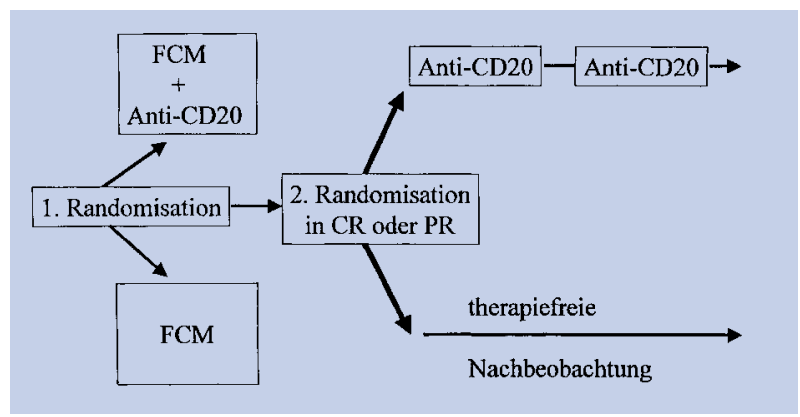

Abb. 2. Therapie im Rezidiv oder bei initialem Therapieversagen.

Remission für jüngere Patienten zu ermitteln. Patienten, die sich in erster Remission nicht für eine myeloablative Radio-Chemotherapie qualifizieren bzw. 60 Jahre oder älter sind, werden ebenfalls wie bisher für eine StandardIFN- $\alpha$-Erhaltung (beginnend mit $3 \times 5$ Mio. E/Woche) versus intensivierte IFN- $\alpha$-Erhaltung (beginnend mit 3 Mio. $\mathrm{E} / \mathrm{Tag}$ ) randomisiert. Ziel dieses Studienabschnitts ist es, die unterschiedlichen IFN- $\alpha$-Erhaltungsstrategien in bezug auf Remissionsdauer und Verträglichkeit zu beurteilen.

In der Gruppe der rezidivierten oder therapierefraktären Patienten soll als initiale Chemotherapie das FCM-Schema, bestehend aus Fludarabin $\left(25 \mathrm{mg} / \mathrm{m}^{2}\right.$ Tage $\left.1-3\right)$, Cyclophosphamid (200 mg/m² Tage 1-3) und Mitoxantron $\left(8 \mathrm{mg} / \mathrm{m}^{2}\right.$ Tag 1$)$, eingesetzt werden. Auch diese Patienten werden initial für eine alleinige Chemotherapie mit FCM versus FCM + Anti-CD20 randomisiert (Abb. 2). Ziel dieses Studienabschnitts ist es wiederum, eine signifikante Verbesserung der initialen Zytoreduktion durch Anti- 
CD20 nachzuweisen. Bei Patienten, die mindestens eine 50\%-Reduktion der initialen Lymphommasse aufweisen, soll des weiteren randomisiert die Möglichkeit einer Erhaltungstherapie mit dem Anti-CD20-Antikörper geprüft werden. Hierzu werden die Patienten nach erfolgreichem Abschluß der initialen Therapie, d.h. nach Erreichen einer kompletten oder partiellen Remission, erneut für eine zweimalige Gabe des Anti-CD20-Antikörpers versus therapiefreie Nachbeobachtung randomisiert. Zielvariable ist für diesen Studienabschnitt das progressionsfreie Überleben der Patienten nach initialer Therapie.

Mit diesen Konzepten verfolgt die Deutsche Studiengruppe Niedrig Maligne Lymphome hochattraktive und vielversprechende Strategien. Sie lassen die Hoffnung berechtigt erscheinen, in naher Zukunft zu einer wesentlichen Verbesserung der bislang noch immer unbefriedigenden Behandlungsergebnisse dieser Erkrankungen beizutragen.

Studienzentrale:

Prof. Dr. W. Hiddemann

Dr. M. Unterhalt

Studienzentrale

Medizinische Klinik III, Klinikum Großhadern

Tegernseer Landstr. 243, D-81549 München

\section{Randomisierte Phase-III-Studie zur Therapie niedrig-maligner Non- Hodgkin-Lymphome in fortgeschrit- tenen Stadien: Chemotherapie vs. Chemotherapie plus Rituximab ${ }^{1}$}

\section{Herold, Erfurt}

Bei dieser Phase-III-Studie handelt es sich um einen prospektiv randomisierten Vergleich einer Kombination des Anti-CD20-Antikörpers Rituximab mit einer MCPChemotherapie gegen eine alleinige MCP-Chemotherapie bei nicht vorbehandelten fortgeschrittenen, behandlungs-

\footnotetext{
${ }^{1}$ Studie der Ostdeutschen Studiengruppe Hämatologie/Onkologie (OSHO). Klinische Prüfung der Hoffmann-LaRoche AG (M 39023). Leiter der klinischen Prüfung: PD Dr. M. Herold, Erfurt.
}

bedürftigen CD-20-positiven niedrig-malignen NonHodgkin-Lymphomen. Die sehr guten, in Phase-I- und -IIStudien erzielten Ergebnisse mit einer alleinigen Rituximab-Therapie bei rezidivierten und resistenten niedrigmalignen, insbesondere follikulären Lymphomen und vor allem die ausgezeichneten Remissions- und Überlebensdaten bei Kombination mit einer CHOP-Chemotherapie rechtfertigen den Einsatz des Antikörpers auch schon in der Primärtherapie im Rahmen dieser klinischen Prüfung.

\section{Einschlußkriterien}

- Therapiebedürftige CD20-positive follikuläre Keimzentrumslymphome (G1 und G2), Stadien III und IV;

- Therapiebedürftige CD20-positive Immunozytome (CD5-negativ), Stadien III und IV;

- CD20-positive Mantelzell-Lymphome, Stadien III und IV;

- Histologische Untersuchung von repräsentativem Material (LK, KM, extranodale Läsion) innerhalb von

6 Monaten vor Studieneinschluß, obligate Referenzpathologie;

- Alter > 18 Jahre und < 75 Jahre;

- Keine zytostatische oder radiologische Vorbehandlung;

- Allgemeinzustand WHO/ECOG $\leq 2$;

- Effektive Kontrazeption;

- Negativer Schwangerschaftstest bei Frauen in gebärfähigem Alter;

- Schriftliches Einverständnis des Patienten nach Aufklärung.

Kriterien zur Therapiebedürftigkeit bei FCL und IC:

- Hämatopoetische Insuffizienz entsprechend BinetStadium C;

- B-Symptomatik;

- Extranodaler Befall (E);

- Bulky-Disease (Lymphome > 7,5 cm, Mediastinaltumor $>1 / 3$ des Thoraxdurchmessers in Höhe Th 5/6;

- Rasches Tumorwachstum (Verdoppelungszeit $<6$ Monate);

- Immunhämatologische Phänomene (AIHA, ITP).

\section{Ausschlußkriterien}

- Möglichkeit einer kurativen Strahlentherapie bei FCL im Stadium III;

- Sekundäres NHL;

- Gleichzeitige Teilnahme an einer anderen klinischen 
Prüfung mit zytostatischer Therapie oder Zytokinen;

- Vorbehandlung mit murinen Antikörpern oder Hypersensitivität gegenüber murinem Eiweiß;

- Begleiterkrankungen bzw. eingeschränkte Organfunktionen, die eine studiengerechte Therapiedurchführung ausschließen;

- Schwangerschaft oder Stillzeit;

- HIV-Positivität;

- Vorausgegangene Organtransplantation.

\section{Randomisierung}

Zentral über die Clinical Research Organisation, ausschließlich via Fax.

\section{Behandlungsprotokoll}

Zur Remissionsinduktion sind 6 Zyklen der Chemotherapie bzw. der Rituximab- + Chemotherapie vorgesehen, dabei wird nach 2 Zyklen ein Zwischenstaging durchgeführt und über die Fortsetzung der Therapie anhand des Remissionsgrades entschieden. Patienten, die nach 6 Zyklen der jeweiligen Behandlung eine komplette oder partielle Remission zeigen, erhalten zwei weitere Konsolidierungszyklen. Bei Fortbestehen der CR oder PR nach der Konsolidierung wird in beiden Studienarmen bei Patienten mit FCL eine Erhaltungstherapie mit Interferon-alpha $(3 \times 4,5$ Mio. E s.c. wöchentlich) ohne zeitliche Begrenzung durchgeführt. Dosisreduktionen der Chemotherapie bei auftretender Myelotoxizität erfolgen nach den allgemein üblichen Richtlinien, eine Dosisreduktion bei Rituximab erfolgt nicht.

Vor der Rituximab-Infusion wird eine Prämedikation mit Paracetamol und Fenistil durchgeführt, die Gabe von $100 \mathrm{mg}$ Prednisolon ist im ersten Behandlungskurs obligat, in den späteren Zyklen fakultativ vorgesehen.

Behandlungsarm A: $M C P$

\begin{tabular}{llll} 
& Dosis, $\mathrm{mg} / \mathrm{m}^{2}$ & Verabreichung & Tage \\
\hline Mitoxantron & 8 & Inf. & $1+2$ \\
Chlorambucil & $3 \times 3$ & p.o. & $1-5$ \\
Prednisolon & 25 & p.o. & $1-5$ \\
\hline Wiederholung alle 4 Wochen. & & \\
\hline
\end{tabular}

Behandlungsarm B: Rituximab $+M C P$

\begin{tabular}{llll}
\hline & Dosis, $\mathrm{mg} / \mathrm{m}^{2}$ & Verabreichung & Tage \\
\hline Rituximab & 375 & $4-5$ h Inf. & 1 \\
Mitoxantron & 8 & Inf. & $3+4$ \\
Chlorambucil & $3 \times 3$ & p.o. & $3-7$ \\
Prednisolon & 25 & p.o. & $3-7$ \\
\hline Wiederholung alle 4 Wochen. & & \\
\hline
\end{tabular}

Flow Sheet: Fortgeschrittene CD20-positive niedrig-maligne NHL mit Therapieindikation (FCL, MCL, IC)

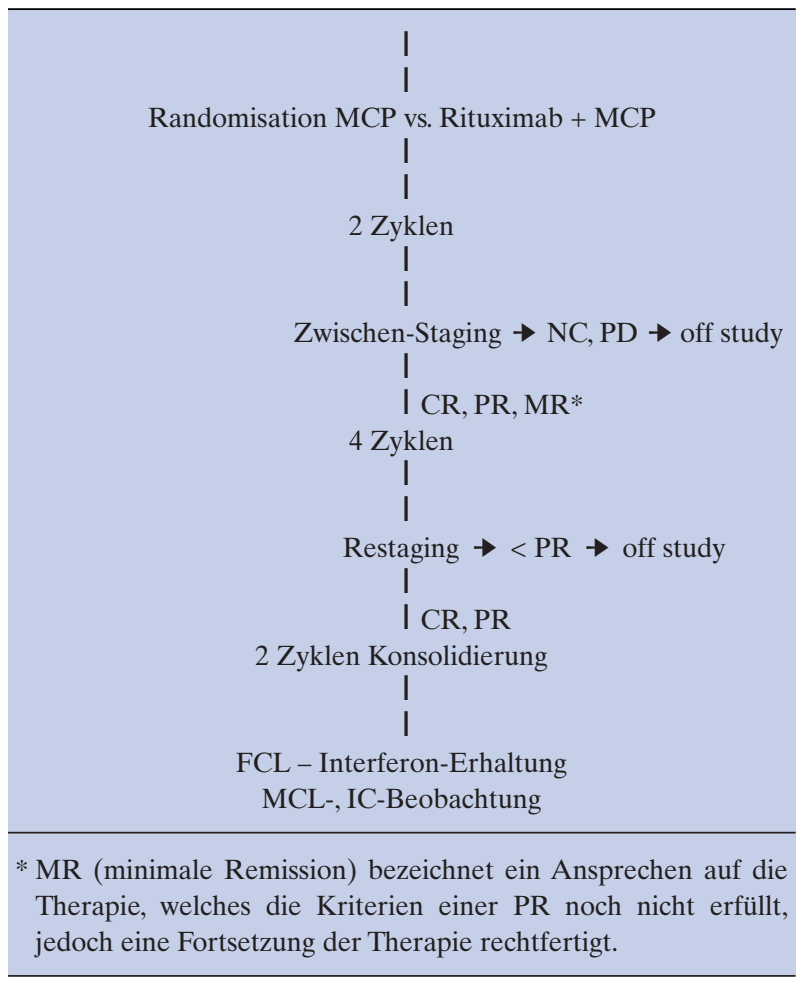

\section{Studienziel}

Studienziel ist der Nachweis einer signifikanten Überlegenheit des experimentellen Therapiearmes, gemessen an den Remissionsraten (CR und PR) und der Freiheit vom Therapieversagen (FFTF), wobei hier nach 4 Jahren ein Unterschied von $20 \%$ erwartet wird. Weitere Prüfziele sind das Gesamtüberleben und die Toxizität. Um zu den genannten Prüfzielen eine statistisch hinreichend sichere 
Aussage zu erhalten, werden insgesamt 310 Patienten rekrutiert, darunter 216 evaluierbare Patienten mit follikulären Keimzentrumslymphomen (Grad 1 und 2); an der klinischen Prüfung nach AMG nehmen insgesamt 34 Behandlungszentren in Kliniken und Schwerpunktpraxen teil. Es ist vorgesehen, die Patientenrekrutierung bis Ende des Jahres 2002 abzuschließen.

Studiensekretariat:

PD Dr. M. Herold

2. Medizinische Klinik, Klinikum Erfurt GmbH

Postfach 595, D-99012 Erfurt

Tel. 0361/781-0, Fax -4803

\section{Randomisierte Studie zur Therapie- optimierung fortgeschrittener niedrig-maligner Non-Hodgkin- und Mantelzell-Lymphome: 2-CdA/Mitoxantron (CdM) vs. Mitoxantron/Chlorambucil/ Prednison (MCP)}

P.S. Mitrou; M.J. Rummel, Frankfurt/M.

\section{Einführung}

Das Purinanalogon Cladribin (2-Chlorodeoxyadenosin, 2-CdA) besitzt eine hohe Aktivität gegen niedrigmaligne Lymphome, die bei vortherapierten und unvorbehandelten Patienten untersucht wurden. In Deutschland wurde die Aktivität der Substanz gegen fortgeschrittene, symptomatische und progrediente niedrig-maligne Lymphome in einer multizentrischen Phase-II-Studie überprüft. Die Ergebnisse wurden zuletzt auf dem ESMOKongreß 1998 in Athen vorgestellt. Die höchste Aktivität wurde beim zentroblastisch-zentrozytischen (cb-cc) und immunozytischen (ic) Lymphom nachgewiesen. 23 von
27 Patienten $(85 \%)$ mit cb-cc-Lymphom sowie 19 von 22 Patienten $(86 \%)$ mit einem Immunozytom sprachen mit einer kompletten oder partiellen Remission an. Die Remissionsrate mit $58 \%$ beim zentrozytischen (cc-)Lymphom war zwar geringer, doch konnte eine therapeutische Aktivität bei dem bekanntermaßen prognostisch sehr ungünstigen cc-Lymphom mit 7 Remissionen bei 12 behandelten Patienten dokumentiert werden. Die Effektivität der Therapie mit 2-CdA war bei nicht vorbehandelten Patienten höher als bei Patienten, die im ersten Rezidiv behandelt wurden; die Rate der kompletten Remissionen lag bei $41 \%$. Abgesehen von der immunsuppressiven Wirkung, charakterisiert durch ausgeprägte Lymphozytopenie sowie Reduktion der CD4+-Zellen im peripheren Blut, war die Nebenwirkungsrate gering und die subjektive Verträglichkeit der Substanz ausgezeichnet.

Weitere klinische Studien müssen der Frage nachgehen, inwiefern 2-CdA in Kombination mit anderen Substanzen, die gegen maligne Lymphome aktiv sind, eine höhere Wirksamkeit bezüglich Remissionsraten und Remissionsdauer $\mathrm{zu}$ erzielen vermag. Bei einer Kombination von 2-CdA mit einer anderen Substanz kann gleichzeitig versucht werden, die Dosis von 2-CdA zu reduzieren, um so eine Verringerung des immunsuppressiven Effekts von 2-CdA zu erzielen. Erste vorläufige Ergebnisse von Studien mit reduzierter Dosis von 2-CdA liegen vor. Mit einer auf $60-70 \%$ reduzierten Dosis von 2-CdA konnte eine vergleichbare Antitumoraktivität bei geringeren Infektionsraten erreicht werden.

Die hohe Wirksamkeit von Mitoxantron in der Behandlung niedrig-maligner Lymphome ist bekannt und untersucht. Zudem ist Mitoxantron als eine relativ gut verträgliche Substanz bekannt, besonders in der Anwendung bei älteren Patienten. Eine Kombination von 2-CdA mit Mitoxantron, die beide eine hohe Aktivität in der Behandlung niedrig-maligner Lymphome und jeweils günstige Nebenwirkungsprofile besitzen, wäre ein sinnvoller Therapieansatz. Eine erste Pilotstudie zur Kombination von 2-CdA mit Mitoxantron wurde von Saven et al. durchgeführt. In eigenen Untersuchungen konnten wir in vitro durch Applikation von 2-CdA in Kombination mit Mitoxantron bei Lymphomzellen von Patienten mit niedrig-malignen Lymphomen einen synergistischen Effekt auf die Induktion von Apoptose beobachten. Diese Ergebnisse stellen eine weitere Rationale für die Kombination von 2-CdA und Mitoxantron dar. Durch das Hinzufügen von Mitoxantron zu 2-CdA kann versucht werden, die Remissionsrate und die Dauer der erzielten Remissionen zu verbessern sowie den immunsuppressiven Effekt von 2-CdA durch eine Dosisreduktion von 2-CdA auf 3 Tage (statt wie bisher 5 Tage) zu verringern. 


\section{Phase-II-Pilotstudie: 2-CdA plus Mitoxantron (CdM)}

Zur Durchführbarkeit der Kombination von 2-CdA und Mitoxantron als Ausgangsstudie für weitere, dann auch prospektiv randomisierte Studienvorhaben wurde diese Kombination in einer Phase-II-Pilotstudie bei Patienten mit niedrig-malignen Non-Hodgkin-Lymphomen als First-Line-Therapie und als Therapie des ersten Rezidivs nach einem vorausgegangenen Behandlungsschema geprüft. Dabei wurde 2-CdA in der Dosierung von $5 \mathrm{mg} / \mathrm{m}^{2}$ als 2-Stunden-Infusion über 3 Tage bei nicht vorbehandelten Patienten mit Mitoxantron $8 \mathrm{mg} / \mathrm{m}^{2}$ an den Tagen 1 und 2 und bei vorbehandelten Patienten mit $12 \mathrm{mg} / \mathrm{m}^{2}$ kombiniert. Erste Ergebnisse dieser Studie wurden auf dem ASCO-Kongreß 1999 in Atlanta vorgestellt. In dieser Studie konnten wir an 64 Patienten zeigen, daß 2-CdA mit Mitoxantron gut kombinierbar ist und eine hohe Aktivität in der Behandlung niedrig-maligner Lymphome aufweist (Tab. 1). Besonders hervorzuheben ist die Ansprechrate von 100\% beim MantelzellLymphom; alle 17 Patienten, davon 8 vorbehandelte, konnten eine Remission erreichen.

Tab. 1. Ergebnisse - CdA / Mitoxantron (CdM)

\begin{tabular}{|c|c|c|c|c|c|c|c|}
\hline \multirow[t]{2}{*}{ Lymphom } & \multirow{2}{*}{$\begin{array}{l}\text { Anzahl } \\
\text { Patienten }\end{array}$} & \multicolumn{2}{|c|}{$\mathrm{CR}$} & \multicolumn{2}{|l|}{ PR } & \multicolumn{2}{|c|}{$\mathrm{CR}+\mathrm{PR}$} \\
\hline & & $\mathrm{n}$ & $\%$ & $\mathrm{n}$ & $\%$ & $\mathrm{n}$ & $\%$ \\
\hline \multicolumn{5}{|l|}{ Follikuläre Keim- } & 36 & 25 & 76 \\
\hline Unbehandelt & 26 & 9 & 35 & 11 & 42 & 20 & 77 \\
\hline Vorbehandelt & 7 & 4 & 57 & 1 & 14 & 5 & 71 \\
\hline Mantelzell-L. & 17 & 6 & 35 & 11 & 65 & 17 & 100 \\
\hline Unbehandelt & 9 & 3 & 33 & 6 & 67 & 9 & 100 \\
\hline Vorbehandelt & 8 & 3 & 38 & 5 & 62 & 8 & 100 \\
\hline Immunozytom & 10 & 2 & 20 & 8 & 80 & 10 & 100 \\
\hline Unbehandelt & 5 & 1 & 20 & 4 & 80 & 5 & 100 \\
\hline Vorbehandelt & 5 & 1 & 20 & 4 & 80 & 5 & 100 \\
\hline MALT/Marginalzell-L. & 3 & 2 & 67 & 1 & 33 & 3 & 100 \\
\hline \multicolumn{8}{|l|}{ Niedrig-malignes } \\
\hline B-Zell-L. & 1 & 1 & & 1 & & 1 & \\
\hline \multicolumn{8}{|l|}{ Alle unbehandelten } \\
\hline B-Zell-L. & 43 & 16 & 37 & 21 & 49 & 37 & 86 \\
\hline \multicolumn{8}{|l|}{ Alle vorbehandelten } \\
\hline B-Zell-L. & 21 & 8 & 38 & 11 & 52 & 19 & 90 \\
\hline Total & 64 & 24 & 38 & 32 & 50 & 56 & 88 \\
\hline
\end{tabular}

\section{Prospektiv randomisierte multizentrische Phase-III-Studie: 2-CdA plus Mitoxantron (CdM) versus Mitoxantron/Chlorambucil/ Prednison (MCP)}

Nur in randomisierten Studien kann der Stellenwert der Kombination von 2-CdA und Mitoxantron (CdM) gegenüber Standardtherapeutika für niedrig-maligne Lymphome (z. B. Chlorambucil, Cyclophosphamid) geprüft werden. Die Kombination aus Mitoxantron, Chlorambucil und Prednison (MCP, oder früher PmM) hat sich in einer randomisierten Studie der Standard-Chemotherapie COP in bezug auf die Induktion kompletter Remissionen sowie auf die Verlängerung des progressionsfreien Überlebens als überlegen erwiesen. Die Therapie mit MCP erscheint als eine sehr wirksame Therapiemodalität und kann als Grundlage und Vergleichstherapiearm zukünftiger Therapiekonzepte herangezogen werden. Im Februar 1999 wurde daher eine prospektiv multizentrische Phase-IIIStudie initiiert mit dem Ziel der randomisierten Prüfung der beiden Therapien CdM versus MCP (Dosierungsschemata in Tab. 2). Primäres Untersuchungsziel der Studie ist die Fragestellung, ob die Therapie mit der Kombination $\mathrm{CdM}$ eine höhere Remissionsrate und ein längeres progressionsfreies Überleben und damit möglicherweise einen Vorteil im Gesamtüberleben im Vergleich zu der Therapiekombination MCP ermöglicht. In die Studie werden Follikelzentrums-Lymphome (follikuläre Lymphome) (cb-cc, zentroblastisch-zentrozytische Lymphome nach der Kiel-Klassifikation), lymphoplasmozytoide Lymphome (Immunozytome) und Mantelzell-Lymphome (cc, zentrozytische Lymphome nach der Kiel-Klassifikation) sowie disseminierte Marginalzonen-Lymphome aufgenommen (Abb. 1). Als Vorbehandlung wird nur eine einmalige Strahlentherapie akzeptiert, eine Vortherapie mit Chemotherapeutika ist ausgeschlossen.

Im Rahmen dieser Studie kann die Prüfmedikation 2-CdA (Cladribin, Leustatin ${ }^{\circledR}$ ) von der Studienzentrale Frankfurt/M. kostenfrei bezogen werden.

Für die zu erwartenden Rezidive der Erkrankung bei Patienten, die bezüglich ihres Alters und ihrer Compliance für eine spätere Rezidiv-Hochdosischemotherapie mit Stammzellsupport in Frage kommen, sollen CD34-positive Stammzellen gesammelt werden, um dann im Rezidiv diese therapeutische Option (Hochdosistherapie mit Stammzellsupport) nutzen zu können. Dazu wird nach dem 3. und unmittelbar vor dem 4. Zyklus ein «Endoxanstoß» (Hochdosis-Cyclophosphamid) mit nachfolgendem G-CSF in der Phthise appliziert, wenn auf CdM oder MCP eine «gute» Remission (CR oder PR > 75\%) erreicht wurde. In der Regenerationsphase sollen dann 
Tab. 2. Behandlungs- und Dosierungsschemata

\begin{tabular}{lllll}
\hline & & Dosis, $\mathrm{mg} / \mathrm{m}^{2}$ & Verabreichung & Tage \\
\hline CdM & 2-CdA & 5 & $\begin{array}{l}\text { 2-Std.-Infusion } \\
\text { i.v. Bolus }\end{array}$ & $1-3$ \\
& Mitoxantron & 8 & & $1+2$ \\
MCP & Chlorambucil & $3 \times 3$ & p.o. & $1-5$ \\
& Mitoxantron & 8 & i.v. Bolus & $1+2$ \\
& Prednison & 25 & p.o. & $1-5$ \\
\hline
\end{tabular}

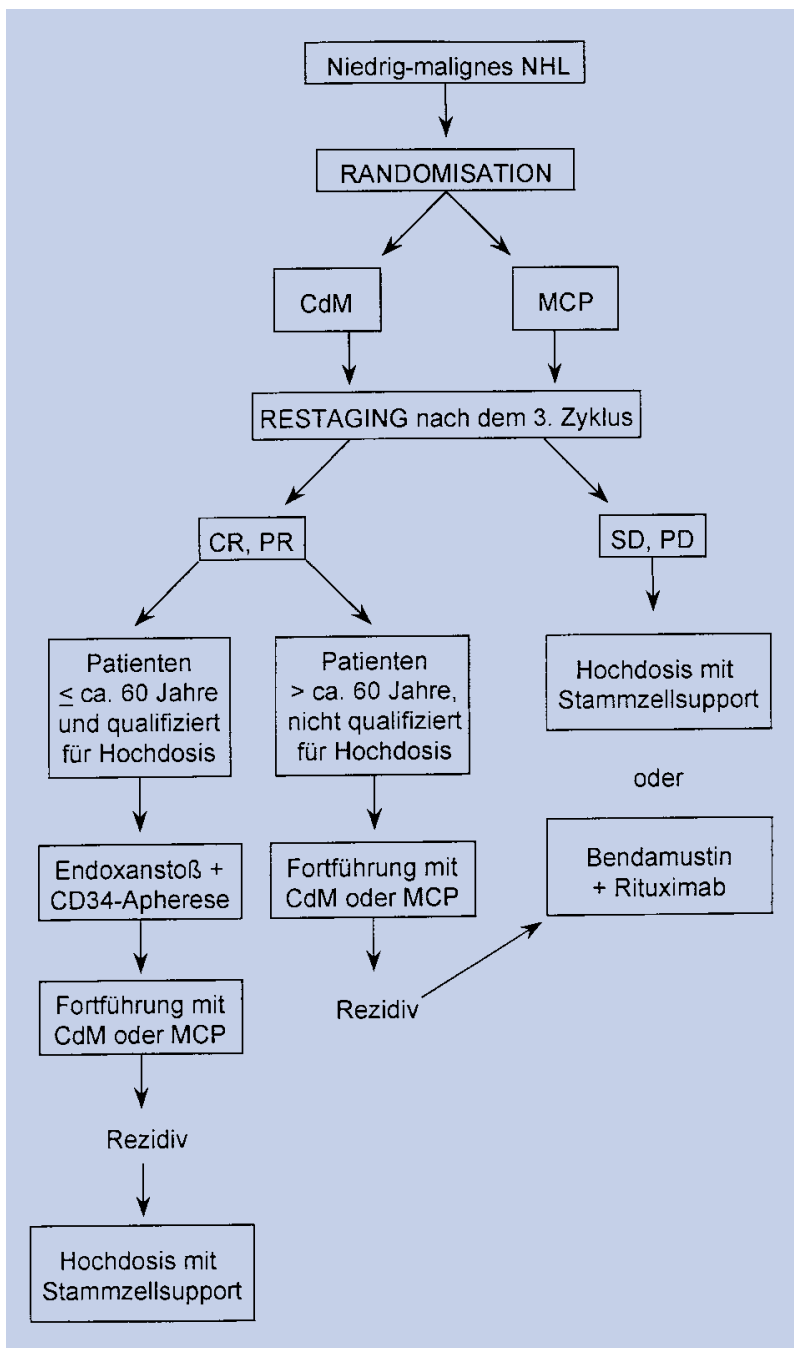

Abb. 1. Niedrig-malignes NHL. CR = Komplette Remission; $\mathrm{PR}=$ partielle Remission; $\mathrm{SD}=$ stable disease $; \mathrm{PD}=$ Progression.
Stammzellen gesammelt werden. Nach erfolgtem Endoxanstoß und Erreichen ausreichender peripherer Blutbildparameter werden die Patienten gemäß ihrem randomisiert zugeteilten Therapiearm (CdM oder MCP) weiterbehandelt. Bei Patienten mit follikulären Lymphomen, die eine Remission erreicht haben, soll eine Erhaltungstherapie mit Interferon-alpha durchgeführt werden. Beim Rezidiv der Erkrankung kann für bis zu etwa 60jährige Patienten eine Hochdosistherapie mit Stammzellsupport durchgeführt werden; Patienten, die älter als 60 Jahre sind oder die für eine Hochdosistherapie nicht in Frage kommen, erhalten Bendamustin, gefolgt von 4 Zyklen Rituximab. Patienten, die auf CdM oder MCP keine Remission erreichen, die also primär therapieresistent sind, können entweder mit Hochdosistherapie und Stammzellsupport oder mit Bendamustin, gefolgt von Rituximab, behandelt werden.

Die Studie, die Anfang dieses Jahres aktiviert wurde, wird noch für etwa 2 Jahre für eine Teilnahme und für den Einschluß von Patienten offen sein. Patienten können für diese Studie in der Studienzentrale per Fax mit einem dafür vorbereiteten Bogen «Patienten-Registrierung» oder per Telefon gemeldet werden:

Studiensekretariat:

Prof. Dr. P. S. Mitrou

Dr. M. J. Rummel

Frau Ilka Muckel (Study Nurse)

Med. Klinik III, Universitätsklinik

Theodor-Stern-Kai 7, D-60590 Frankfurt

Tel. 069/6301-4039, Fax -7373

(Literaturangaben: Auf Anfrage bei den Autoren erhältlich.)

\section{Impressum}

Beilage zu ONKOLOGIE, Band 22, Heft 5, Oktober 1999

Mit freundlicher Unterstützung durch Wyeth-Pharma GmbH, Münster.

Die Wiedergabe von Gebrauchsnamen, Handelsnamen, Warenbezeichnungen usw. in dieser Zeitschrift berechtigt auch ohne besondere Kennzeichnung nicht zu der Annahme, daß solche Namen im Sinne der Warenzeichen- und Markenschutz-Gesetzgebung als frei zu betrachten wären und daher von jedermann benutzt werden dürften. Für Angaben über Dosierungsanweisungen und Applikationsformen kann vom Verlag keine Gewähr übernommen werden. Derartige Angaben müssen vom jeweiligen Anwender im Einzelfall anhand anderer Literaturstellen auf ihre Richtigkeit überprüft werden.

Alle Rechte vorbehalten.

Ohne schriftliche Genehmigung des Verlags dürfen diese Publikation oder Teile daraus nicht in andere Sprachen übersetzt oder in irgendeiner Form mit mechanischen oder elektronischen Mitteln (einschließlich Fotokopie, Tonaufnahme und Mikroskopie) reproduziert oder auf einem Datenträger oder Computersystem gespeichert werden. (c) Copyright 1999 by S. Karger GmbH, Postfach,

D-79095 Freiburg, und S. Karger AG, Postfach, CH-4009 Basel Druck: Walter Biering GmbH, Grafischer Betrieb, München. 